\title{
Virtual reality replays of sports performance: Effects on memory, feeling of competence, and performance
}

\author{
Anne A. Cuperus* ${ }^{*}$, , Ineke J.M. van der Ham ${ }^{1}$ \\ Experimental Psychology, Utrecht University, Heidelberglaan 1, 3584 CS Utrecht, The Netherlands
}

\section{A R T I C L E I N F O}

\section{Article history:}

Received 20 September 2016

Accepted 21 September 2016

\section{Keywords:}

Virtual reality

Misinformation effect

False memories

Memory alteration

Feeling of competence

Sports performance

\begin{abstract}
A B S T R A C T
Memory can be altered after receipt of misleading information; the misinformation effect was first studied almost 40 years ago. Later studies showed that suggestive information could even lead to the creation of new false memories in people. Whereas previous research focused primarily on false information about passively observed events, we aimed to investigate whether memory for one's own physical performance can be altered by means of false, manipulated replays of these events in virtual reality, displayed from a first person viewpoint. We further explored the possibility of using the misinformation effect beneficially, by investigating whether it can affect feeling of competence, as well as subsequent sports performance. Participants $(\mathrm{N}=27)$ took four series of shots at a goalpost on a soccer field. Between these series, they were shown three different types of virtual reality replays of their performance; one accurate representation of actual performance and two manipulated versions, one that made performance seem worse (negative manipulation) and one that made performance seem better (positive manipulation). Participants rated their feeling of competence before and after each replay and rated how accurately the replay displayed their real-life shots at the goal. The manipulated replays were considered equally accurate representations of actual performance as the non-manipulated ones. Also, the type of replay manipulation positively correlated with feeling of competence but did not influence sports performance. The present study showed that memory for one's own physical performance can be altered by means of manipulated virtual reality replays and that this can be used beneficially.
\end{abstract}

(C) 2016 Elsevier Inc. All rights reserved.

\section{Introduction}

For quite some time now it has been known that retrieving memories is a reconstructive process (Bartlett, 1932). Unlike a video recording, which is the same when played repeatedly, information retrieval from human memory is susceptible to errors. Memory may be altered after receipt of misleading information; the first study on this 'misinformation effect' was conducted almost 40 years ago by Loftus, Miller, and Burns (1978). Studies showed that false details can be planted into memory for both simulated (e.g., a filmed car accident), as well as real-world events (Loftus, 2005). Moreover, the way questions about a past event are formulated can even alter memory for it. For instance, when asked how fast cars were going in films of automobile accidents, participants reported higher estimates of speed when the question contained the verb

\footnotetext{
* Corresponding author.

E-mail address: acuperus@gmail.com (A.A. Cuperus).

1 Present address: Health, Medical and Neuropsychology, Leiden University, Wassenaarseweg 52, 2333 AK Leiden, The Netherlands.
} 


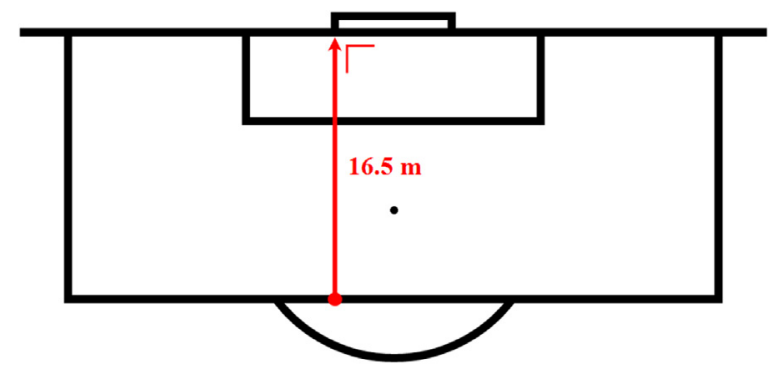

Fig. 1. The resting position of the ball during the sport task in the experiment (red dot). (For interpretation of the references to colour in this figure legend, the reader is referred to the web version of this article.)

'smashed' than when the same question contained the verbs 'collided', 'bumped', 'contacted', or 'hit' in place of 'smashed' (Loftus \& Palmer, 1974). Such findings stress the importance of using proper questioning techniques in court testimonies (Powell, 2005).

Later studies showed that suggestive information could even lead to the creation of new false memories in people. False memories were implanted by means of various forms of media, such as a written narrative about one's childhood (Loftus \& Pickrell, 1995) or a doctored photograph (Wade, Garry, Read, \& Lindsay, 2002). These studies can be framed in terms of varying levels of 'media richness' (Segovia \& Bailenson, 2009), which can be described based on four criteria: capacity for immediate feedback; capacity to transmit multiple cues such as graphic symbols or human gestures; language variety, including numbers and natural language; capacity of the medium to have a personal focus (Daft, Lengel, \& Trevino, 1987). According to Segovia and Bailenson (2009), the stronger the false, suggestive information is in terms of media richness, the more likely people are to adopt the information into memory. The personal focus criterion may be especially important in this respect, as self-referent encoding yields superior memory (Symons \& Johnson, 1997).

Whereas previous research focused primarily on false information about passively observed events, in the present study we aimed to investigate whether memory for one's own physical performance can be altered by means of false, manipulated replays of these events in virtual reality (VR), displayed from a first-person viewpoint. The inherent characteristics of VR (e.g., user-specific viewpoints and a wide, three-dimensional field of view) provide a great sense of immersion, which makes it a very rich form of media (Segovia \& Bailenson, 2009). We therefore expected that watching the manipulated replays would alter memory, so that the manipulated replays would be considered equally accurate representations of actual performance as the non-manipulated ones. In the present study we further explored the possibility of using the misinformation effect beneficially, by investigating whether the manipulated replays can affect feeling of competence, which refers to the concept of self-efficacy as introduced by Bandura (1977); the extent or strength of one's belief in one's own ability to complete tasks and reach goals. If so, such replays could potentially be used for therapeutic purposes, such as prevention or treatment of performance anxiety or trauma following bad performance in, for example, a soccer match. Furthermore, they may affect subsequent sports performance, as an increased feeling of competence should lead to an increase in intrinsic motivation, which in turn is positively correlated with quality of performance (Ryan \& Deci, 2000).

\section{Method}

\subsection{Participants}

Participants were recruited via coaches of different soccer clubs and through a Facebook advertisement. To be eligible, participants had to be at least 18 years old and have played on a soccer team at a club for at least one year. Twenty-seven male amateur soccer players participated, and their mean age was 23.2 years (range $18-28 ; S D=3.00$ ).

\subsection{Procedure}

After reading the information sheet, participants signed the consent form. According to the information sheet, the goal of the experiment was to test the effects of watching VR replays of one's own sports performance on subsequent performance. Participants were first instructed to carry out a physical sport task, in which they had to take 10 shots across the ground at the left goalpost on a soccer field, from a $16.5 \mathrm{~m}$ distance (Fig. 1).

The experimenter recorded the distance missed with respect to the goalpost, as well as the side of the goalpost along which the ball passed. The distances were read from a tape measure ( $10 \mathrm{~m}$ long), which was rolled out on the goal line. After the sport task, participants were taken to the side of the field. Here, they rated their feeling of competence by indicating how difficult they considered the sport task, and how good they considered themselves in carrying out the task, on two $100 \mathrm{~mm}$ visual analogue scales (VAS) that ranged from 0 (very hard/very bad) to 100 (very easy/very good). Meanwhile, the experimenter entered the recorded miss distances and sides of the goalpost into the VR application (a modification of "Beyond Sports" v0.53; developed by Triple). When the competence test was finished, participants were instructed to put 


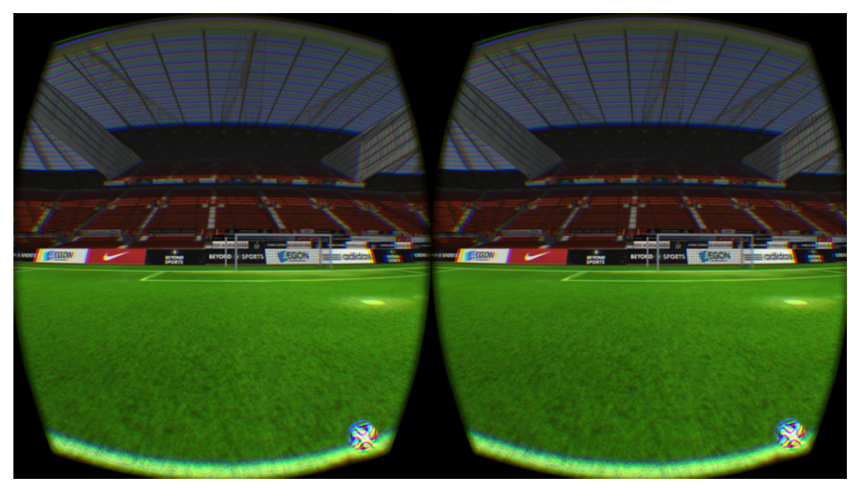

Fig. 2. Monitor display during a VR replay.

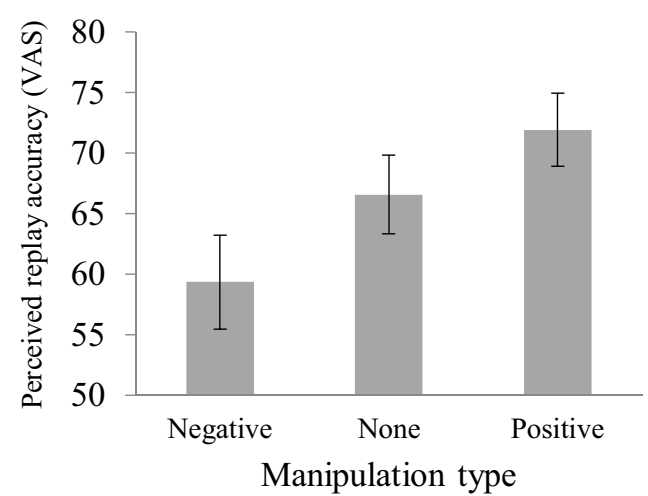

Fig. 3. Mean VAS (0; very inaccurately - 100; very accurately) scores on perceived replay accuracy for manipulated and non-manipulated VR replays. The error bars represent standard errors.

on the head-mounted display (Oculus Rift Development Kit 2), through which the first VR replay of their performance on the sport task was shown for all 10 shots (Fig. 2).

The VR replays could either be an accurate representation of the actual performance or a manipulated version thereof. In the manipulations the distance between the ball and the goalpost was adjusted, making the performance seem worse (miss distances multiplied by 1.5 ; negative manipulation) or better (miss distances multiplied by 0.5 ; positive manipulation) than the actual performance. The VR application contained no 'hit detection' system, so if in reality the ball hit the goalpost or the net, the replay would show the ball going through it.

After the replay, participants rated their feeling of competence a second time, as well as how accurately the replay displayed their real-life shots at the goal on a single $100 \mathrm{~mm}$ VAS that ranged from 0 (very inaccurately) to 100 (very accurately). The sequence from the sport task to the replay accuracy rating was repeated two more times, so that all three types of VR replays were shown. The order in which the replays were offered was counterbalanced among participants. Finally, the sport task was carried out one last time. Afterwards, participants were informed about the actual goal of the experiment, and were told in which order the different types of replays were shown. The total procedure took approximately $40 \mathrm{~min}$.

\subsection{Data analyses}

Scores on all measures (perceived replay accuracy, feeling of competence, and sports performance) were analyzed by repeated measures ANOVAs with manipulation type (negative vs. none vs. positive) as a within-subjects factor.

\section{Results}

Fig. 3 shows mean scores on perceived replay accuracy, for each type of manipulation. Mauchly's test indicated that the assumption of sphericity was violated $\left(\chi^{2}(2)=6.09, p<0.05\right)$; therefore, degrees of freedom were corrected using Greenhouse-Geisser estimates of sphericity $(\varepsilon=0.82)$. The repeated measures ANOVA showed that perceived replay accuracy was significantly influenced by type of manipulation, $F(1.65,42.76)=5.52, p<0.05, \eta_{\mathrm{p}}^{2}=0.18$. 


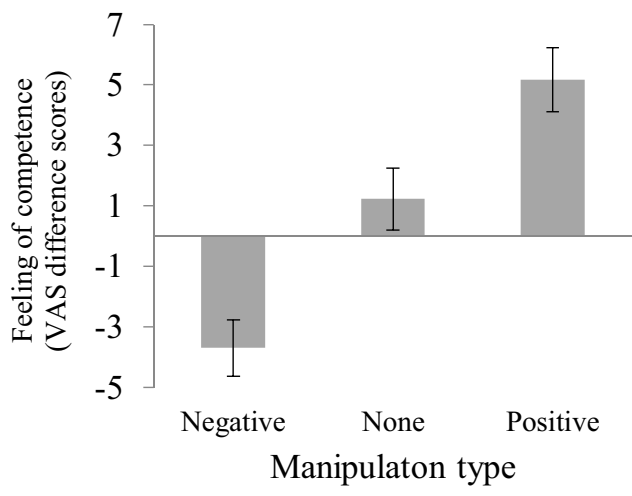

Fig. 4. Mean VAS ( 0 ; very hard/very bad - 100; very easy/very good) difference scores on feeling of competence following manipulated and non-manipulated VR replays. The error bars represent standard errors.

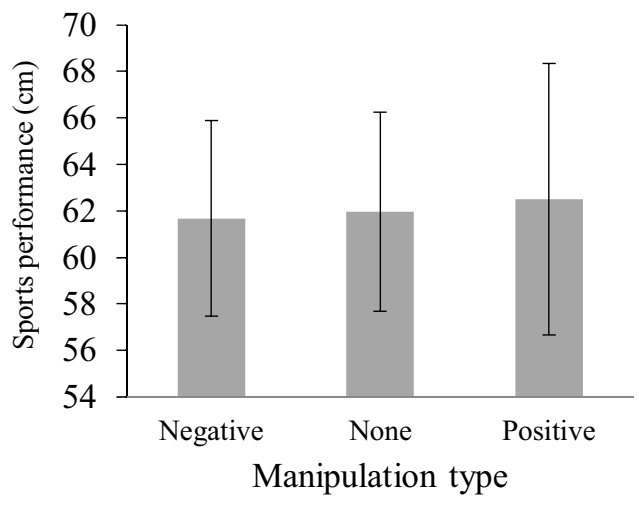

Fig. 5. Mean distance missed $(\mathrm{cm})$ on the sport task after watching manipulated and non-manipulated VR replays. The error bars represent standard errors.

Post-hoc Bonferroni corrected $t$-tests showed that positive manipulations were rated as significantly more accurate representations of performance than negative manipulations, $p=0.03$. Also, a trend showed that non-manipulated replays were rated as more realistic than negative manipulations, $p=0.07$.

Fig. 4 illustrates changes (pre vs. post VR replay) in feeling of competence, for each type of manipulation. The repeated measures ANOVA showed that feeling of competence was significantly influenced by type of manipulation, $F(2,52)=26.46$, $p<0.01, \eta_{\mathrm{p}}^{2}=0.50$.

Post-hoc Bonferroni corrected $t$-tests showed that the increase in feeling of competence after watching positive manipulations differed significantly from the decrease after watching negative manipulations, $p<0.01$. The same conclusion applies to watching non-manipulated replays compared to negative manipulations, $p<0.01$. Finally, the increase in feeling of competence after watching positive manipulations was also significantly greater than the increase after seeing non-manipulated replays, $p<0.05$.

Fig. 5 shows sports performance, measured in mean distance missed $(\mathrm{cm})$, for each type of VR replay. Mauchly's test indicated that the assumption of sphericity was violated $\left(\chi^{2}(2)=7.43, p<0.05\right)$; therefore, degrees of freedom were corrected using Greenhouse-Geisser estimates of sphericity $(\varepsilon=0.80)$. The repeated measures ANOVA showed that sports performance was not significantly influenced by type of manipulation, $F(1.59,41.37)<1$.

\section{Discussion}

Whereas previous research focused primarily on false information about passively observed events, in the present study we aimed to investigate whether memory for one's own physical performance can be altered by means of false, manipulated replays of these events in virtual reality (VR), displayed from a first person viewpoint. The inherent characteristics of VR (e.g., user-specific viewpoints and a wide, three-dimensional field of view) provide a great sense of immersion, which makes it a very rich form of media (Segovia \& Bailenson, 2009). We therefore expected that watching the manipulated replays would alter memory, so that the manipulated replays would be considered equally accurate representations of actual performance as the non-manipulated ones. In the present study we further explored the possibility of using the misinformation effect beneficially, by investigating whether it can affect feeling of competence, as well as subsequent sports performance. 


\subsection{Memory alteration}

As expected, the manipulated replays were considered equally accurate representations of actual performance as the nonmanipulated ones. However, the positive manipulations were considered more realistic than the negative manipulations, and a similar trend was found for the positive manipulations in comparison to the non-manipulated replays. It could be caused by a 'self-serving bias', which can be described as any cognitive or perceptual process that is distorted by the need to maintain and enhance self-esteem (Sherrill, 2008). In the present study, participants may have rejected the negative manipulations by rating them as less realistic, thereby maintaining their self-esteem. Also, people generally have a tendency to distort their memories in a positive way (Loftus, 1982). The positive manipulations may have confirmed the correctness of such 'prestige-enhancing' memories, leading to higher ratings for these replays.

\subsection{Feeling of competence and sports performance}

The type of replay manipulation positively correlated with feeling of competence; negative manipulations decreased participants' feeling of competence, whereas positive manipulations increased it. As the replays are a form of feedback on personal sports performance, this finding fits nicely with previous sports research, which showed that coach feedback is positively correlated with feeling of competence (Allen \& Howe, 1998). We suggest it could be worth exploring whether manipulated VR reconstructions of memories can be used for therapeutic purposes, such as prevention or treatment of performance anxiety or trauma following bad performance in a soccer match or other sports competition.

According to self-determination theory (Ryan \& Deci, 2000), an increased feeling of competence leads to an increase in intrinsic motivation, which in turn is positively correlated with quality of performance. Manipulation type however did not influence sports performance in the present study. Perhaps this lack of an effect on performance was caused by the fact that only amateur soccer players participated, who were not able to translate changes in feeling of competence into better or worse performance due to a lack of skill. Another explanation could be that the effect of feeling of competence on motivation was too small. The strength of the relationship between feeling of competence and motivation to perform should be addressed in further research.

\section{Acknowledgements}

This study was conducted in collaboration with the Beyond Sports department of Triple (Alkmaar, the Netherlands). IH was supported by a Veni grant (451-12-004) from the Netherlands Organization for Scientific Research (NWO).

\section{References}

Allen, J. B., \& Howe, B. L. (1998). Player ability, coach feedback, and female adolescent athletes' perceived competence and satisfaction. Journal of Sport E Exercise Psychology, 20, 280-299.

Bandura, A. (1977). Self-efficacy: Toward a unifying theory of behavioral change. Psychological Review, 84, 191-215. http://dx.doi.org/10.1037/0033-295X.84.2.191

Bartlett, F. C. (1932). Remembering: An experimental and social study. Cambridge: Cambridge: University.

Daft, R. L., Lengel, R. H., \& Trevino, L. K. (1987). Message equivocality, media selection, and manager performance: Implications for information systems. Management Information Systems Quarterly, 11, 355-366. http://dx.doi.org/10.2307/248682

Loftus, E. F., \& Palmer, J. C. (1974). Reconstruction of automobile destruction: An example of the interaction between language and memory. Journal of Verbal Learning and Verbal Behavior, 13, 585-589. http://dx.doi.org/10.1016/S0022-5371(74)80011-3

Loftus, E. F., \& Pickrell, J. E. (1995). The formation of false memories. Psychiatric Annals, 25, 720-725. http://dx.doi.org/10.3928/0048-5713-19951201-07

Loftus, E. F., Miller, D. G., \& Burns, H. J. (1978). Semantic integration of verbal information into a visual memory. Journal of Experimental Psychology: Human Learning and Memory, 4, 19-31. http://dx.doi.org/10.1037//0278-7393.4.1.19

Loftus, E. F. (1982). Memory and its distortions. In A. G. Kraut (Ed.), G. Stanley Hall lectures (pp. 119-154). Washington, DC: American Psychological Association.

Loftus, E. F. (2005). Planting misinformation in the human mind: A 30-year investigation of the malleability of memory. Learning E' Memory, 12, 361-366. http://dx.doi.org/10.1101/1m.94705

Powell, M. B. (2005). Improving the reliability of child witness testimony in court: The importance of focusing on questioning techniques. Current Issues in Criminal Justice, 17, 137-151.

Ryan, R. M., \& Deci, E. L. (2000). Self-determination theory and the facilitation of intrinsic motivation, social development, and well-being. American Psychologist, 55, 68-78. http://dx.doi.org/10.1037//0003-066X.55.1.68

Segovia, K. Y., \& Bailenson, J. N. (2009). Virtually true: Children’s acquisition of false memories in virtual reality. Media Psychology, 12, 371-393. http://dx.doi.org/10.1080/15213260903287267

Sherrill, M. (2008). Self-serving bias. In W. A. Darity (Ed.), International Encyclopedia of the Social Sciences. Detroit: Macmillan Reference USA, pp. 429.

Symons, C. S., \& Johnson, B. T. (1997). The self-reference effect in memory: A meta-analysis. Psychological Bulletin, 121, $371-394$. http://dx.doi.org/10.1037/0033-2909.121.3.371

Wade, K. A., Garry, M., Don Read, J., \& Lindsay, D. S. (2002). A picture is worth a thousand lies: Using false photographs to create false childhood memories. Psychonomic Bulletin E Review, 9, 597-603. http://dx.doi.org/10.3758/BF03196318 